\title{
Magnetic fields in irregular galaxies
}

\section{Amanda Kepley ${ }^{1} \dagger$, Eric Wilcots ${ }^{1}$, Ellen Zweibel ${ }^{1}$, Stefanie Mühle ${ }^{2}$,} John Everett ${ }^{1}$, Timothy Robishaw ${ }^{3}$, Carl Heiles ${ }^{4}$, and Uli Klein ${ }^{5}$

${ }^{1}$ Department of Astronomy, University of Wisconsin-Madison, 475 N. Charter St., Madison, WI 53704, USA

email: kepley@virginia.edu,ewilcots@astro.wisc.edu,zweibel@astro.wisc.edu, everett@physics.wisc.edu

${ }^{2}$ Stichting JIVE, Postbus 2, 7990 AA Dwingeloo, The Netherlands email: muehle@jive.nl

${ }^{3}$ School of Physics, The University of Sydney, NSW 2006, Australia email: robishaw@physics.usyd.edu.au

${ }^{4}$ Astronomy Department, University of California, Berkeley, California 94720-3411, USA email: heiles@astro.berkeley.edu

${ }^{5}$ Argelander-Institut für Astronomie, Universität Bonn, Auf dem Hügel 71, D-53121 Bonn, Germany

email: uklein@astro.uni-bonn.de

\begin{abstract}
The low masses of irregular galaxies change the behavior of their interstellar medium (ISM) compared to that of normal spirals, so the role of magnetic fields in the ISM in irregulars may be very different than in spirals. We present high-resolution and high-sensitivity observations of the magnetic fields of two irregular galaxies: NGC 4214 and NGC 1569.
\end{abstract}

Keywords. Magnetic fields - polarization - ISM: magnetic fields - galaxies: dwarf - galaxies: ISM - galaxies: irregular - galaxies: magnetic fields - galaxies: starburst - radio continuum: galaxies

The chaotic interstellar medium (ISM) of irregular galaxies is very different from ISM of spirals. Irregulars rotate as solid bodies and their ISM is prone to disruption because of their low masses. What role does the magnetic field play in the ISM of these galaxies? To date only five irregulars have detailed observations of their magnetic fields (NGC 4449: Chyży et al. 2000; IC 10 and NGC 6822: Chyży et al. 2003; LMC: Klein et al. 1993, Gaensler et al. 2005; SMC: Mao et al. 2008). Our goal is to increase the number of irregulars with observed magnetic fields to address the following questions: 1) what generates and sustains large-scale magnetic fields in irregulars and 2) what causes the range of magnetic field structure seen in irregulars? Here we present observations of two well studied, actively star-forming irregular galaxies: NGC 1569 and NGC 4214.

For NGC 4214, we estimate from the $6 \mathrm{~cm}$ synchrotron flux that the field strength is $50 \mu \mathrm{G}$ in the center and $18 \mu \mathrm{G}$ at the edges. The magnetic pressure is comparable to the pressures of the other ISM components and thus does not dominate the ISM. We do not detect much polarization, implying that the field is random. To see if an $\alpha-\omega$ dynamo could generate large-scale field in NGC 4214, we calculated the dynamo number using an HI rotation curve (Allsopp 1979). It is above the critical value only for radii of 2 to 4 kpc, so NGC 4214 is not able to produce a large-scale field over its entire disk with an $\alpha-\omega$ dynamo.

$\dagger$ Present address: Department of Astronomy, University of Virginia, P.O. Box 400325, Charlottesville, VA 22904-4325 


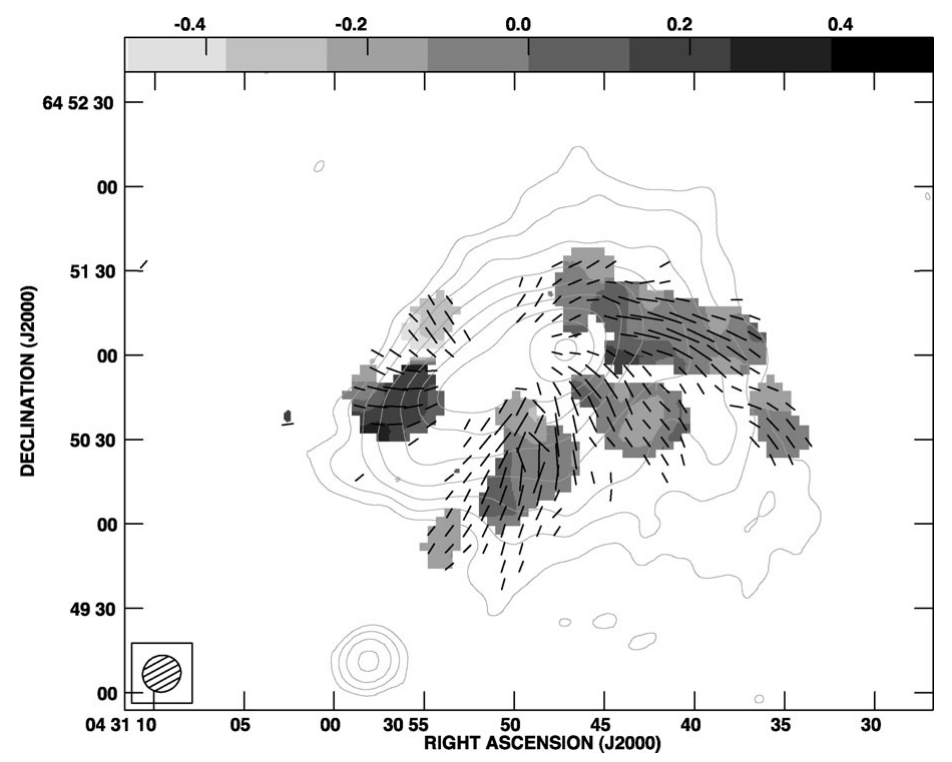

Figure 1. The magnetic field structure of NGC 1569 (Kepley et al. 2009, in preparation). The contours show the $3 \mathrm{~cm}$ radio continuum emission, the pseudo-vectors show the magnetic field orientation, and the greyscale shows the rotation measures. The contours are $3,6,12,24,48$, $96,192,384$, and 768 times $29 \mu \mathrm{Jy}_{\text {beam }}{ }^{-1}$, a pseudo-vector with a length of $1^{\prime}$ has a polarized intensity of $12.8 \mu \mathrm{Jy}_{\text {beam }}^{-1}$, and the rotation measures are in units of $1000 \mathrm{rad} \mathrm{m}^{-2}$.

NGC 1569 is one of the most extreme starbursts in the local universe. This highly inclined galaxy has a strong outflow (Martin 1998, Westmoquette et al. 2008). Its magnetic field strength, estimated from the $3 \mathrm{~cm}$ synchrotron flux, ranges from $35 \mu \mathrm{G}$ in the center to $10 \mu \mathrm{G}$ at the edges. The magnetic pressure is comparable to the pressure of the $\mathrm{X}$-ray gas, so the field does not dominate the ISM. The field in the disk is mostly random, which reflects the turbulent ISM there. The plane of sky halo field is roughly perpendicular to the disk. The polarized regions are correlated with $\mathrm{H} \alpha$ bubbles. The observed field has a bubble-like morphology, e.g. the southwestern most region of polarization points away from the observer near the center of the galaxy, but is mostly in the plane of the sky in the halo of the galaxy. These structures are analogous to the $\alpha-\omega$ dynamo model of $\mathrm{K}$. Ferrière, e.g., Ferrière \& Schmitt (2000).

\section{References}

Allsopp, N. J. 1979, MNRAS 188, 765

Chyży, K. T., Beck, R., Kohle, S., Klein, U., \& Urbanik, M. 2000, A $₫ A$ 355, 128

Chyży, K. T., Knapik, J., Bomans, D. J., Klein, U., Beck, R., Soida, M., \& Urbanik, M. 2003, A $\& A 405,513$

Ferrière, K. \& Schmitt, D. 2000, A\& A 358, 125

Gaensler, B. M., Haverkorn, M., Staveley-Smith, L., Dickey, J. M., McClure-Griffiths, N. M., Dickel, J. R., \& Wolleben, M. 2005, Science 307, 1610

Klein, U., Haynes, R. F., Wielebinski, R., \& Meinert, D. 1993, A\&A 271, 402

Mao, S. A., Gaensler, B. M., Stanimirovic, S., Haverkorn, M., McClure-Griffiths, N. M., StaveleySmith, L., \& Dickey, J. M. 2008, arXiv:0807.1532

Martin, C. L. 1998, ApJ 506, 222

Westmoquette, M. S., Smith, L. J., \& Gallagher, J. S. 2008, MNRAS 383, 864 